\title{
Antimicrobial assesment of aroylhydrazone derivatives in vitro
}

\author{
SAŠA POLOVIĆ \\ VANJA LJOLJIĆ BILIĆ ${ }^{2}$ \\ ANA BUDIMIR ${ }^{3}$ \\ DARKO KONTREC 4 \\ NIVES GALIĆ $5^{*}$ \\ IVAN KOSALEC ${ }^{*}$ \\ ${ }^{1}$ Agency for Medicinal Products \\ and Medical Devices of Croatia \\ Zagreb, Croatia \\ ${ }^{2}$ Department of Microbiology \\ Faculty of Pharmacy and Biochemistry \\ University of Zagreb \\ Zagreb, Croatia \\ ${ }^{3}$ Department of General and Inorganic \\ Chemistry, Faculty of Pharmacy and \\ Biochemistry, University of Zagreb \\ Zagreb, Croatia \\ ${ }^{4}$ Department of Organic Chemistry \\ and Biochemistry, Ruđer Bošković \\ Institute, PO Box 180, Zagreb, Croatia \\ ${ }^{5}$ Department of Chemistry \\ Faculty of Science \\ University of Zagreb, Zagreb, Croatia
}

Accepted December 14, 2018

Published online February 11, 2019

\begin{abstract}
Aroylhydrazones 1-13 were screened for antimicrobial and antibiofilm activities in vitro. $N^{\prime}$-(2-hydroxy-phenylmethylidene)-3-pyridinecarbohydrazide (2), $N^{\prime}$-(5-chloro-2-hydroxyphenyl-methylidene)-3-pyridinecarbohydrazide (10), $N^{\prime}-(3,5-$ chloro-2-hydroxyphenylmethylidene)-3-pyridinecarbohydrazide (11), and N'-(2-hydroxy-5-nitrophenylmethylidene)-3pyridinecarbohydrazide (12) showed antibacterial activity against Escherichia coli, with MIC values (in $\mu \mathrm{mol} \mathrm{mL} \mathrm{m}^{-1}$ ) of $0.18-0.23,0.11-0.20,0.16-0.17$ and $0.35-0.37$, resp. Compounds 11 and 12, as well as $N^{\prime}$-(2-hydroxy-3-methoxyphenylmethylidene)-3-pyridinecarbohydrazide (6) and $N^{\prime}$-(2-hydroxy-5methoxyphenylmethylidene)-3-pyridinecarbohydrazide (8) showed antibacterial activity against Staphylococcus aureus, with the lowest MIC values of $0.005-0.2,0.05-0.12,0.06-0.48$ and $0.17-0.99 \mu \mathrm{mol} \mathrm{mL} \mathrm{m}^{-1}$. $N^{\prime}$-(2-hydroxy-5-methoxyphenylmethylidene)-3-pyridinecarbohydrazide (7) showed antifungal activity against both fluconazole resistant and susceptible C. albicans strains with $I C_{90}$ range of $0.18-0.1 \mu \mathrm{mol} \mathrm{mL} \mathrm{m}^{-1}$. Only compound $\mathbf{1 1}$ showed activity against C. albicans ATCC 10231 comparable to the activity of nystatin (the lowest MIC $4.0 \times 10^{-2}$ vs. $\left.1.7 \times 10^{-2} \mu \mathrm{mol} \mathrm{mL} \mathrm{m}^{-1}\right)$. Good activity regarding multi-resistant clinical strains was observed for compound $\mathbf{1 2}$ against MRSA strain (MIC $0.02 \mu \mathrm{mol} \mathrm{mL} \mathrm{mL}^{-1}$ ) and compounds 2, 6 and 12 against ESBL+ E. coli MFBF 12794, with the lowest MIC for compound $12\left(I_{50} 0.16 \mu \mathrm{mol} \mathrm{mL}{ }^{-1}\right)$. Anti-biofilm activity was found for compounds 2 (MBFIC 0.015-0.02 $\mu \mathrm{mol} \mathrm{mL} \mathrm{mL}^{-1}$ against MRSA) and 12 (MBFIC $0.013 \mu \mathrm{mol} \mathrm{mL}-1$ against EBSL+ E. coli). In the case of compound 2 against MRSA biofilm formation, $M B F I C$ values were comparable to those of gentamicin sulphate, whereas in the case of compound $\mathbf{1 2}$ and EBSL+E. coli even more favourable activity compared to gentamicin was observed.
\end{abstract}

Keywords: aroylhydrazones, antimicrobial, antibiofilm, MDR strains

Aroylhydrazones contain an azomethine group $-\mathrm{NHN}=\mathrm{CH}-$ connected with carbonyl group, which is responsible for their different pharmaceutical applications. Therefore, aroylhydrazones have attracted considerable attention for their wide range of biological

\footnotetext{
*Correspondence, ngalic@chem.pmf.hr; ikosalec@pharma.hr
} 
activities, such as antimicrobial, anticonvulsant, antitumor, analgesic, antiplatelet, antitubercular and anti-inflammatory activities $(1,2)$. Further, hydrazones as chelating agents were investigated as potential drugs for the treatment of a disease called "iron overload disease" (3). Salicylaldehyde derivatives were shown to be selective iron chelators with promising pharmacological properties (4). However, pharmacokinetic studies have stressed the relatively short biological half-life of certain hydrazones due to hydrolysis of the hydrazone bond, which is typical for this class of compounds $(4,5)$. Molecular structural changes of aroylhydrazones derived from salicylaldehyde, $O$-vanilin and nicotinic acid hydrazide in dimethylsulphoxide/water (DMSO/water) mixtures were studied by NMR, UV-Vis, ATR and Raman spectroscopy (6). Results obtained by Galić et al. (6) revealed that addition of water to the system did not induce tautomeric conversion of the ketoamino hydrazide part and the enolimino aldehyde part. Furthermore, addition of water showed formation of hydrated molecules and compounds were quite stable in DMSO/ water mixtures (V(DMSO)/ $V\left(\mathrm{H}_{2} \mathrm{O}\right)$, of $9 / 1,8 / 2,7 / 3$ and $6 / 4$ volume ratios).

According to the work done by Da Costa et al. (7), in vitro evaluated $N$-acylhydrazone derivatives of different amino acids such as L-phenylalanine, L-leucine and L-alanine against $M$. tuberculosis showed a MIC between $12.5-50 \mu \mathrm{g} \mathrm{mL} \mathrm{m}^{-1}$. In another study, done by Mandewale et al. (8), zinc(II) complexes with quinoline hydrazone ligands were synthesized and screened for activity against M. tuberculosis (H37 RV strain) ATCC 27294. Results showed that two of the tested complexes were very active, with MIC value ranging from 8.00-7.42 $\mu \mathrm{mol} \mathrm{L}^{-1}$, which is comparable to the drugs ciprofloxacin (MIC value $9.41 \mu \mathrm{mol}$ $\mathrm{L}^{-1}$ ), pyrazinamide (MIC value $25.34 \mu \mathrm{mol} \mathrm{L}^{-1}$ ) and streptomycin (MIC $10.74 \mu \mathrm{mol} \mathrm{L}^{-1}$ ) used to treat tuberculosis.

Benzimidazole derivatives bearing hydrazone moiety revealed that some of the compounds had a bactericidal effect on the growth of Salmonella typhimurium, two times better or equal to the activity of chloramphenicol, which was used as a positive control (9). Activities of these compounds against other Gram-negative bacterial strains such as Escherichia coli, Proteus vulgaris, Klebsiella pneumoniae or Pseudomonas aeruginosa were good, with the MIC value of 25-100 $\mathrm{mg} \mathrm{mL}^{-1}$. Activities against Gram-positive bacteria were determined on four strains: Listeria monocytogenes, Staphylococcus aureus, Enterococcus faecalis and Bacillus subtilis. The best activity, equal to the activity of chloramphenicol, was found against an $E$. faecalis strain with the MIC value of $12.5 \mu \mathrm{g} \mathrm{mL} \mathrm{m}^{-1}$.

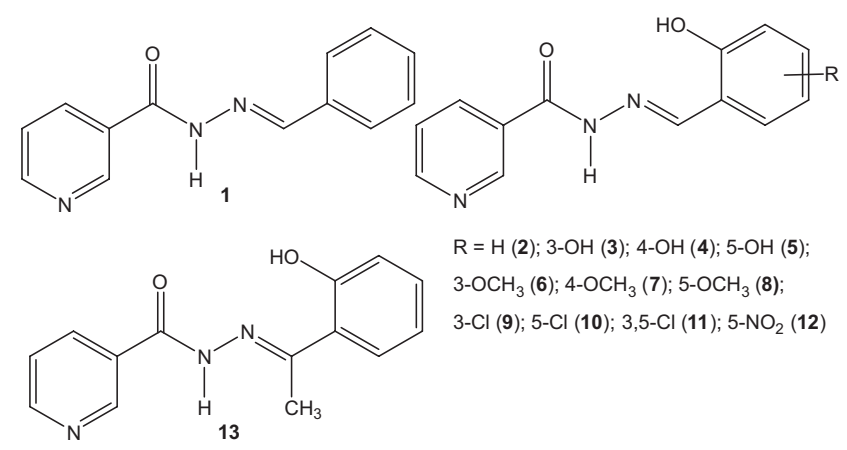

Fig. 1. Structures of aroylhydrazones 1-13. 
As a part of our investigations on aromatic hydrazones derived from nicotinic acid hydrazide (10), a group of 13 derivatives (Fig. 1) was studied for antimicrobial activity in the present work. Our research was focused on antibacterial, antifungal and antibiofilm studies in vitro.

\section{EXPERIMENTAL}

\section{Chemicals}

Chemicals used in this study, such as acetic acid glacial (Panreac, Spain), dimethyl sulfoxide pure (Lach-Ner, Czech Republic), methanol (Merck KgaA, Germany), are commercially available and were used without further purification, while aroylhydrazone derivatives were synthesized by previously described procedures (11) and analyzed using standard analytical methods. Drugs used as positive controls were gentamicin sulphate and colistin purchased from Sigma-Aldrich, nystatin from PLIVA Hrvatska (Croatia), norfloxacin from Krka-Farma (Slovenia) and voriconazole from Pfizer (USA).

\section{Microorganisms and media}

Several microorganisms, standard laboratory strains from the American Type Culture Collection (ATCC) and resistant clinical strains, were obtained from stock cultures of the Collection of Microorganisms (MFBF), Department of Microbiology, Faculty of Pharmacy and Biochemistry, University of Zagreb, Croatia. Experiments included Staphylococcus aureus ATCC 6538 (methilicin susceptible strain), methicillin resistant S. aureus MFBF 10679 (MRSA), Escherichia coli ATCC 10536, extended-spectrum beta-lactamase-positive E. coli (ESBL+ E. coli) MFBF 12794, Candida albicans ATCC 10231 (fluconasole-susceptible), fluconasole-resistant C. albicans MFBF $11103\left(\mathrm{Ca}^{\mathrm{FLR}+}\right)$, and Aspergillus brasiliensis ATCC 16404 . Microbiological media Mueller-Hinton broth, Mueller-Hinton+2 \% glucose $(\mathrm{m} / \mathrm{V})$ broth and RPMI $1640+2 \%(m / V)$ glucose broth were purchased from Merck (Germany).

\section{Antimicrobial susceptibility testing}

Minimum inhibitory concentrations (MICs) were measured by the serial two-fold microdilution broth assay in Mueller-Hinton broth for bacterial strains, and RPMI with $2 \%$ of glucose broth for fungi according to the recommendations of EUCAST protocols $(12,13)$.

Compounds 1-13 were first dissolved in DMSO/water (1:1, V/V), followed by dilution with sterile physiological saline to a stock concentration of $800 \mu \mathrm{g} \mathrm{mL} \mathrm{L}^{-1}$. The solvent mixture was tested as a negative control in all assays.

Inoculum suspensions of bacteria and fungi were prepared from fresh cultures of microbial strains cultured on the surface of tryptic-soy agar for $18 \mathrm{~h}$ at $35^{\circ} \mathrm{C}$ for bacterial species, and on Sabouraud $2 \%(\mathrm{~m} / \mathrm{V})$ glucose agar for $48 \mathrm{~h}$ at $35^{\circ} \mathrm{C}$ for yeast and fungi. Inocula were prepared with physiological saline and cell density was adjusted to $0.5 \mathrm{Mc}-$ Farland units using a nephelometer (Kisker, Germany). Working microbial suspensions were prepared as 1:10 dilutions in Mueller-Hinton broth for bacteria, and RPMI-2 \% glucose broth for yeast and fungi. Final bacterial suspensions contained approximately $10^{7}$ $\mathrm{CFU} \mathrm{mL} \mathrm{m}^{-1}$ and the yeast/mold suspensions $5 \times 10^{6} \mathrm{CFU} \mathrm{mL}^{-1}$. Serial two-fold microdilution 


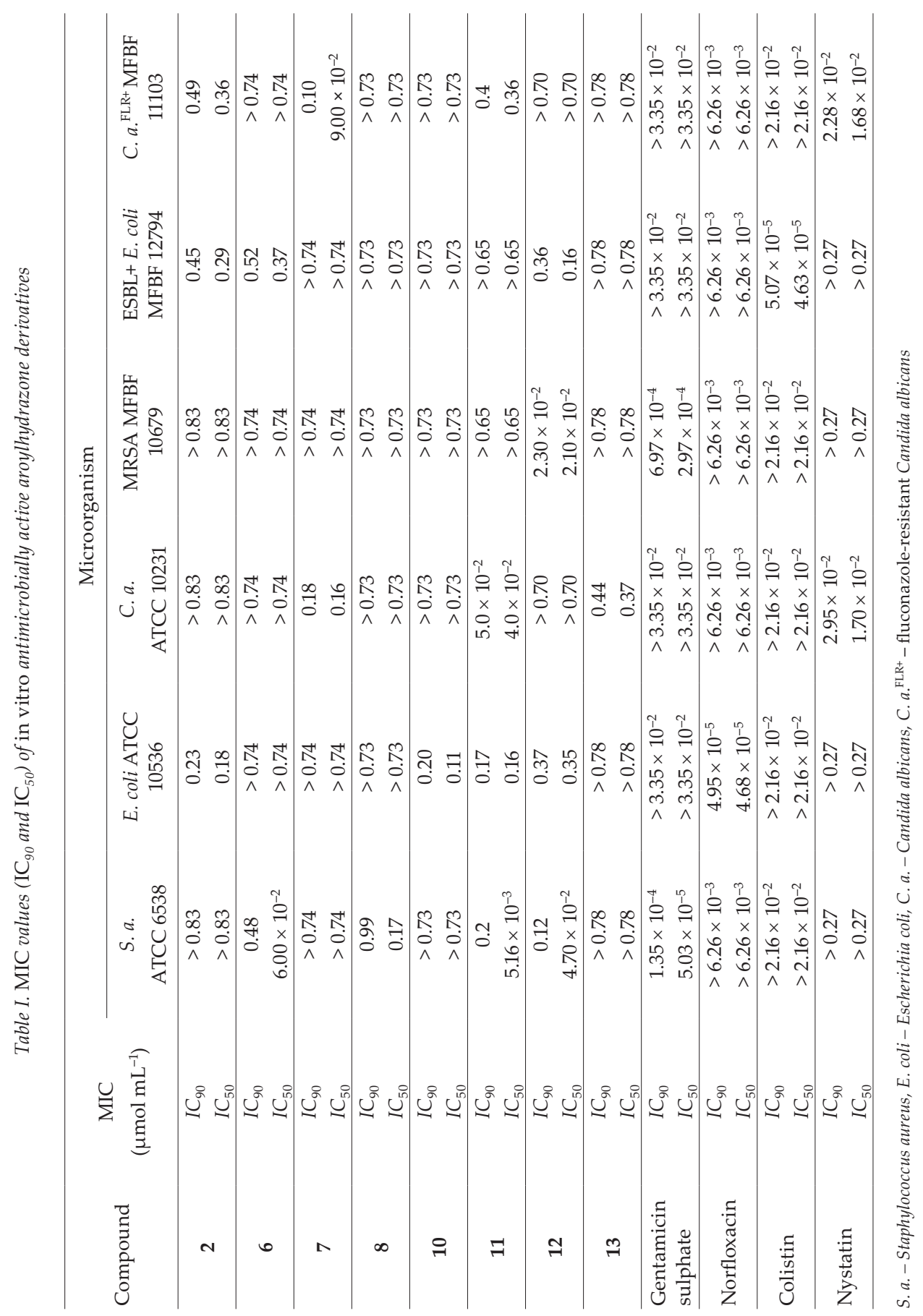


testing was performed for all compounds in concentrations from 200 to $0.1 \mu \mathrm{g} \mathrm{mL} \mathrm{L}^{-1}$. After inoculation and aerobic incubation for $18 \mathrm{~h}$ at $35{ }^{\circ} \mathrm{C}$ in the dark, MICs of bacterial strains were determined by adding the redox-indicator 2,3,5-triphenyl-2 $\mathrm{H}$-tetrazolium chloride [TTC, $1.0 \%(\mathrm{~m} / \mathrm{V})$, in sterile physiological saline]. After 3 hours of incubation at $35{ }^{\circ} \mathrm{C}$ in the dark, $0.04 \mathrm{~mol} \mathrm{~L}^{-1} \mathrm{HCl}$ in isopropanol was added to all wells and absorbance was recorded at $540 \mathrm{~nm}$ using a microtiter plate reader (Labsystems iEMS Reader MF, Finland). MIC was defined as the lowest concentration of investigated compounds that inhibited $90 \%$ of the growth compared to the negative control; values for $50 \%$ growth inhibition are shown as well. MICs for C. albicans strains were obtained by determing absorbance at $540 \mathrm{~nm}$ using the above mentioned microtiter plate reader. MIC values for mold A. brasiliensis ATCC 16404 were determined visually after $48 \mathrm{~h}$ of incubation, aerobically at $35^{\circ} \mathrm{C}$ in the dark. MICs were calculated using the Gompertz non-linear regression model, evaluating the \% microbial viability vs. $\log _{\text {conc }}$ (GraphPad Prism version 6.00 for Windows, GraphPad Software, La Jolla California USA, www.graphpad.com) to get the final $I C_{90}$ and $I C_{50}$ values expressed in $\mu \mathrm{g} \mathrm{mL}^{-1}$. All tests were performed in triplicate and results are expressed as the mean value. Minimal inhibitory concentrations are shown in Table I.

\section{Determination of anti-biofilm activity}

Compounds that showed antimicrobial activity against the tested clinical microbial strains MRSA MFBF 10679, ESBL+ E. coli MFBF 12794 and Ca ${ }^{\mathrm{FLR}+}$ MFBF 11103 were investigated for anti-biofilm activity using the crystal violet assay according to Vlainić et al. (14) with slight modifications. The effect on biofilm formation was evaluated by determining the minimum biofilm formation inhibition concentration (MBFIC). Inoculum suspensions of fresh cultures of microbial strains were prepared with sterile physiological saline and cell density was adjusted to $0.5 \mathrm{McF}$ arland units using a nephelometer (Kisker). Working suspensions of bacteria and yeast used in experiments were prepared as 1:10 dilutions in RPMI 1640+2 \% $(\mathrm{m} / \mathrm{V})$ glucose broth for yeast, and Mueller-Hinton $+2 \%$ glucose $(\mathrm{m} / \mathrm{V})$ broth for bacteria. Final bacterial suspensions contained $10^{7} \mathrm{CFU} \mathrm{mL} \mathrm{m}^{-1}$ and yeast suspensions 5 $\times 10^{6} \mathrm{CFU} \mathrm{mL} \mathrm{m}^{-1}$. Experiments were performed in sterile 96-well flat-bottom plastic tissue plates (TPP, Switzerland). Investigated compounds were tested in the concentration range from 500 to $3.91 \mu \mathrm{g} \mathrm{mL} \mathrm{m}^{-1}$. Untreated controls contained inoculum in broth, and standard antimicrobial drugs were used for positive controls - gentamicin sulphate (stock concentration used for ESBL+ E. coli MFBF 12794 was $1 \mathrm{mg} \mathrm{mL}^{-1}$, while stock concentration for MRSA MFBF 10679 was $600 \mu \mathrm{g} \mathrm{mL}^{-1}$ ), norfloxacin (stock concentration of $1 \mathrm{mg} \mathrm{mL}^{-1}$ ) for bacteria, nystatin and voriconazole (stock concentration of $400 \mu \mathrm{g} \mathrm{mL} \mathrm{m}^{-1}$ each) for fungi. Only broth was used for negative controls. After a 24-h incubation period for bacteria and $48 \mathrm{~h}$ of incubation for yeast $\left(37^{\circ} \mathrm{C}\right.$, aerobic, in the dark), wells were aspirated and washed four times with $250 \mu \mathrm{L}$ of PBS and shaken vigorously. Adherent microbial cells were treated with methanol for $20 \mathrm{~min}$ and left to dry overnight. The formed biofilm was stained with crystal violet $(0.5 \%, \mathrm{~m} / \mathrm{V}$, in methanol) for $10 \mathrm{~min}$. Residual crystal violet was aspirated and plates were rinsed with tap water and left to dry overnight. Adherent biofilm was resolubilized with glacial acetic acid. Results were obtained by measuring absorbance at $540 \mathrm{~nm}$ using a microtiter plate reader (Labsystems iEMS Reader MF). The $M B F I C_{50}$ and $M B F I C_{90}$ values represent the lowest compound dilutions at which microbial growth during biofilm formation was inhibited by 50 and $90 \%$, resp., compared to the untreated control (inoculum with broth). MBFICs were calculated from linear regression of the log 
of concentration of compounds vs. \% reduction, using the GraphPad Prism version 6.00 for Windows, GraphPad Software, La Jolla California USA, www.graphpad.com. All tests were performed in triplicate and the results are expressed as the mean value.

\section{RESULTS AND DISCUSSION}

All the compounds 1-13 were screened for their in vitro antibacterial activity against S. aureus (Gram-positive) and E. coli (Gram-negative) strains, C. albicans (yeast) and $A$. brasiliensis (filamentous fungus).

Susceptibility of the tested microbial species to aroylhydrazone compounds showed species-dependent and compound-dependent activity. Pronounced activities were detected for compounds 2, 6, 7, 8, 10, 11, 12 and 13. Compounds 2, 6, 7, 11 and 12 showed good activity against multi-resistant clinical strains MRSA, ESBL+ E. coli and C. $a{ }^{\text {FLR+ }}$, still lower than that of reference drugs. The same applies to compounds $\mathbf{1 1}$ and 12, which were highly active against S. aureus ATCC 6538 (both 11 and 12), C. albicans ATCC 10231 (11) and E. coli ATCC 10536 (11) but still not as active as the standard drugs. Compound $\mathbf{6}$ showed activity against the $S$. aureus ATCC 6538 strain with $I C_{90}$ range $6.0 \times 10^{-2}-0.48 \mu \mathrm{mol} \mathrm{mL}^{-1}$, while compounds $\mathbf{1 1}$ and $\mathbf{1 2}$ showed even better antibacterial activity against $S$. aureus, with the

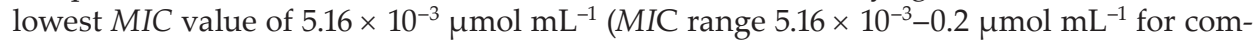
pound 11) as well as against the E. coli ATCC 10536 strain $\left(I C_{90}\right.$ range $\left.0.17-0.37 \mu \mathrm{mol} \mathrm{mL}^{-1}\right)$. Compound 2 showed antibacterial activity against E. coli with the MIC range 0.18-0.23 $\mu \mathrm{mol} \mathrm{mL} \mathrm{m}^{-1}$, whereas compound $\mathbf{1 0}$ showed enhanced activity against $E$. coli with the MIC range $0.11-0.20 \mu \mathrm{mol} \mathrm{mL} \mathrm{m}^{-1}$. In all of these cases, MIC values were markedly higher than for standard drugs. Good activity against both investigated C. albicans strains was shown by compound 11. The lowest MIC value of $4.0 \times 10^{-2} \mu \mathrm{mol} \mathrm{mL}^{-1}$ against $C$. albicans ATCC 10231 was fairly comparable with the lowest MIC value of nystatin of $1.7 \times 10^{-2} \mu \mathrm{mol} \mathrm{mL}^{-1}$. Compound 7 showed somewhat lower activity against both C. albicans strains with higher MIC values $\left(I C_{90}\right.$ range $\left.0.18-0.10 \mu \mathrm{mol} \mathrm{mL} \mathrm{m}^{-1}\right)$. Only compound 12 showed good activity against the MRSA strain, but still markedly lower than gentamicin sulphate. Compounds 2, 6 and 12 showed activity against ESBL+E. coli MFBF 12794, with the lowest MIC in the case of

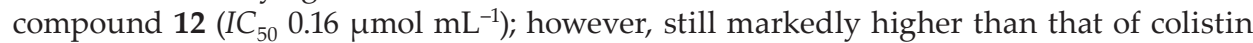
$\left(I C_{50} 4.63 \times 10^{-5} \mu \mathrm{mol} \mathrm{mL} L^{-1}\right)$.

None of the tested compounds showed a wide spectrum of antibacterial activity against multi-resistant clinical strains and none of the compounds showed activity against A. brasiliensis in the concentration range tested.

Antibacterial tests performed in this study showed that compounds $\mathbf{1 0}$ and 11, bearing one and two chloro atoms, resp., as well as compound $\mathbf{1 2}$ with a nitro group, showed good inhibition of bacterial growth whereas the compounds without an electron withdrawing group (chloro- and nitro-) showed weak antimicrobial activity. This may be due to the fact that electron withdrawing substituents (chloro-, nitro-) increase the lipophilicity of the compounds, which leads to higher partitioning of such compounds into the lipophilic phase of a microbial membrane. This causes higher local concentration of the compound near a biological target site (1). Furthermore, hydrazones with a strong electron withdrawing substituent (fluorine) showed high bioactivity and were commonly used in medicine to improve metabolic stability, bioavailability and protein ligand interactions (15). 
Table II. $\mathrm{MBFIC}_{50}$ and $\mathrm{MBFIC}_{90}$ values of in vitro antimicrobially active aroylhydrazone derivatives

\begin{tabular}{|c|c|c|c|}
\hline \multirow{2}{*}{ Compound } & \multirow{2}{*}{$\begin{array}{c}\text { MBFIC } \\
\left(\mu \mathrm{mol} \mathrm{mL} L^{-1}\right)\end{array}$} & \multicolumn{2}{|c|}{ Microorganism } \\
\hline & & MRSA MFBF 10679 & ESBL+ E. coli MFBF 12794 \\
\hline \multirow{2}{*}{2} & $M_{B F I C}{ }_{50}$ & $1.54 \times 10^{-2}$ & $>2.07 \times 10^{-2}$ \\
\hline & MBFIC $_{90}$ & $2.03 \times 10^{-2}$ & $>2.07 \times 10^{-2}$ \\
\hline \multirow{2}{*}{12} & $M_{B F I C}{ }_{50}$ & $>1.75 \times 10^{-2}$ & $1.32 \times 10^{-2}$ \\
\hline & $M{ }^{2} F I C_{90}$ & $>1.75 \times 10^{-2}$ & $1.34 \times 10^{-2}$ \\
\hline \multirow{2}{*}{$\begin{array}{l}\text { Gentamicin } \\
\text { sulphate }\end{array}$} & MBFIC $_{50}$ & $1.17 \times 10^{-2}$ & $>0.17$ \\
\hline & $M_{B F I C} C_{90}$ & $1.36 \times 10^{-2}$ & $>0.17$ \\
\hline \multirow{2}{*}{ Norfloxacin } & $\mathrm{MBFIC}_{50}$ & $>0.78$ & $>0.78$ \\
\hline & $M_{B F I C}{ }_{90}$ & $>0.78$ & $>0.78$ \\
\hline
\end{tabular}

According to the experimental results, none of the compounds under investigation showed anti-biofilm activity of C. $a .{ }^{\mathrm{FLR}+}$ at the tested concentrations. As shown in Table II, anti-biofilm activity of compounds $\mathbf{2}$ and $\mathbf{1 2}$ was detected in the case of MRSA and EBSL + E. coli. Compound $\mathbf{2}$ showed good activity only against the formation of biofilm formed by MRSA, while compound $\mathbf{1 2}$ showed good activity only against the formation of biofilm formed by EBSL $+E$. coli. In the case of compound 2 against MRSA biofilm formation, MBFIC values $\left(1.5 \times 10^{-2} \mu \mathrm{mol} \mathrm{mL} L^{-1}-2.0 \times 10^{-2} \mu \mathrm{mol} \mathrm{mL}^{-1}\right)$ were comparable to those of gentamicin sulphate $\left(1.2 \times 10^{-2} \mu \mathrm{mol} \mathrm{mL}-1.4 \times 10^{-2} \mu \mathrm{mol} \mathrm{mL} \mathrm{mL}^{-1}\right)$, whereas in the case of compound 12 and EBSL+ E. coli even more favourable activity versus gentamicin was recorded. Results reveal that the type of substituent in the molecule that determines hydrophobicity/hydrophilicity of the hydrazone could be affecting quorum sensing systems (QS) involved in anti-biofilm activity and, therefore, in future, further investigation of this issue should be performed.

Compounds containing electron withdrawing groups generally exert better antimicrobial activity against Gram-negative bacteria. These findings can be explained by the differences between Gram-negative and Gram-positive bacteria. Gram-negative bacteria are surrounded by a thin peptidoglycan cell wall, which itself is surrounded by an outer membrane containing lipopolysaccharide. Gram-positive bacteria lack an outer membrane but are surrounded by layers of peptidoglycan many times thicker than those found in Gram-negative bacteria. Nitro group is a strong electron-withdrawing group, situated in para position to a hydroxyl group in compound 12, which leads to increased lipophilicity. Increased lipophilicity promotes entrance of substances into the cell membrane and causes structural defects leading to cell death. As already mentioned, compound $\mathbf{1 2}$ (nitro substituted) showed high activity against the formation of a biofilm formed by the Gram-negative bacterium (EBSL+ E. coli).

\section{CONCLUSIONS}

All the title compounds were screened for their in vitro antimicrobial activity against S. aureus (Gram-positive bacterium), E. coli (Gram-negative bacterium), C. albicans and A. 
brasiliensis. The activity was detected for the compounds $N^{\prime}$-(2-hydroxy-phenylmethylidene)3-pyridinecarbohydrazide (2), N'-(2-hydroxy-3-methoxyphenylmethylidene)-3-pyridinecarbohydrazide (6), $N^{\prime}$-(2-hydroxy-5-methoxyphenylmethylidene)-3-pyridinecarbohydrazide (7), N'-(2-hydroxy-5-methoxyphenylmethylidene)-3-pyridinecarbohydrazide (8), $N^{\prime}$-(5-chloro-2-hydroxyphenylmethylidene)-3-pyridinecarbohydrazide (10), N'-(3,5-chloro-2-hydroxyphenylmethylidene)-3-pyridinecarbohydrazide (11), and $N^{\prime}$-(2-hydroxy5-nitrophenylmethylidene)-3-pyridinecarbohydrazide (12). Compounds 11 and 12 showed antibacterial activity against S. aureus and E. coli strains, while compounds $\mathbf{6}$ and $\mathbf{8}$ showed activity only against $S$. aureus. Compounds 2 and 10 were active against $E$. coli. Experimental results reveal that compounds 6, 8, 11 and 12 exhibited strong antibacterial activity against $S$. aureus. As the obtained results show, only compound $\mathbf{1 2}$ showed strong activity against MRSA. Furthermore, only compound $\mathbf{1 1}$ showed activity against $C$. albicans ATCC 10231 comparable to the activity of nystatin.

Compounds 2, 6, 10, 11 and 12, which showed good antimicrobial activity, were also investigated on multi-resistant clinical strains MRSA, ESBL+ E. coli and C. $a .{ }^{\mathrm{FLR}+}$. Experimental results showed that only compounds $\mathbf{2}$ and $\mathbf{1 2}$ showed good activity against biofilm formation. Compound 2 showed good activity against formation of the biofilm formed by MRSA, while compound $\mathbf{1 2}$ showed only good activity against formation of the biofilm formed by EBSL $+E$. coli. In the case of compound 2 against MRSA biofilm formation, $M B F I C$ values were comparable to those of gentamicin sulphate, whereas in the case of compound 12 and EBSL+ E. coli even more favourable activity compared to gentamicin was observed.

Acknowledgements. - The research was supported by the Croatian Science Foundation (Project IP-2014-09-4841).

\section{REFERENCES}

1. Ł. Popiołek, Hydrazide-hydrazones as potential antimicrobial agents: overview of the literature since 2010, Med. Chem. Res. 26 (2017) 287-301; DOI 10.1007/s00044-016-1756-y

2. M. K. Dahlgren, C. E. Zetterström, Å. Gylfe, A. Linusson and M. Elofsson, Statistical molecular design of a focused salicylidene acylhydrazide library and multivariate QSAR of inhibition of type III secretion in the Gram-negative bacterium Yersinia, Bioorg. Med. Chem. 18 (2010) 2686-2703; https://doi.org/10.1016/j.bmc.2010.02.022

3. P. V. Bernhardt, P. Chin, P. C. Sharpe and D. R. Richardson, Hydrazone chelators for the treatment of iron overload disorders: iron coordination chemistry and biological activity, Dalton Trans. 30 (2007) 3232-3244; https://doi.org/10.1039/b704102k

4. K. Hruskova, P. Kovarikova, P. Bendova, P. Haskova, E. Mackova, J. Stariat, A. Vavrova, K. Vavrova and T. Simunek, Synthesis and initial in vitro evaluations of novel antioxidant aroylhydrazone iron chelators with increased stability against plasma hydrolysis, Chem. Res. Toxicol. 24 (2011) 290-302; https://doi.org/10.1021/tx100359t

5. P. Kovaríkova, Z. Mrkvičkova and J. Klimeš, Investigation of the stability of aromatic hydrazones in plasma and related biological material, J. Pharm. Biomed. Anal. 47 (2008) 360-370; https://doi. org/10.1016/j.jpba.2008.01.011

6. N. Galić, A. Dijanošić, D. Kontrec and S. Miljanić, Structural investigation of aroylhydrazones in dimethylsulphoxide/water mixtures, Spectrochim. Acta A 95 (2012) 347-353; https:// doi.org/10.1016/j. saa.2012.03.086 
7. C. F. Da Costa, A. C. Pinheiro, M. V. De Almeida, M. C. Lourenço and M. V. De Souza, Synthesis and antitubercular activity of novel amino acid derivatives, Chem. Biol. Drug Des. 79 (2012) 216222; https://doi.org/10.1111/j.1747-0285.2011.01269.x

8. M. C. Mandewale, B. Thorat, Y. Nivid, R. Jadhav, A. Nagarsekar and R. Yamgar, Synthesis, structural studies and antituberculosis evaluation of new hydrazone derivatives of quinoline and their Zn(II) complexes, J. Saudi Chem. Soc. 22 (2018) 218-228; https://doi.org/10.1016/j.jscs.2016.04.003

9. Y. Ozkay, Y. Tunali, H. Karaca and I. Işikdă̆, Antimicrobial activity and a SAR study of some novel benzimidazole derivatives bearing hydrazone moiety, Eur. J. Med. Chem. 45 (2010) 3293 3298; https://doi.org/10.1016/j.ejmech.2010.04.012

10. T. Benković, A. Kenđel, J. Parlov-Vuković, D. Kontrec, V. Chiş, S. Miljanić and N. Galić, Multiple dynamics of aroylhydrazone induced by mutual effect of solvent and light - spectroscopic and computational study, J. Mol. Liq. 255 (2018) 18-25; https://doi.org/10.1016/j.saa.2017.09.038

11. T. Benković, D. Kontrec, V. Tomišić, A. Budimir and N. Galić, Acid-base properties and kinetics of hydrolysis of aroylhydrazones derived from nicotinic acid hydrazide, J. Solution Chem. 45 (2016) 1227-1245; https://doi.org/10.1007/s10953-016-0504-8

12. European Committee for Antimicrobial Susceptibility Testing (EUCAST) of the European Society for Clinical Microbiology and Infectious Diseases (ESCMID), EUCAST Discussion Document E.Dis 5.1, Determination of minimum inhibitory concentrations (MICs) of antibacterial agents by broth dilution, Clin. Microbiol. Infect. 9 (2003) 1-7; https://doi.org/10.1046/j.1469-0691.2003.00790.x

13. M. C. Arendrup, J. Meletiadis, J. W. Mouton, K. Lagrou, Petr Hamal, J. Guinea, and the Subcommittee on Antifungal Susceptibility Testing (AFST) of the ESCMID European Committee for Antimicrobial Susceptibility Testing, EUCAST Definitive Document E.Def. 7.3.1. January 2017 - Method for the Determination of Broth Dilution Minimum Inhibitory Concentrations of Antifungal Agents for Yeasts; http://www.eucast.org/ast_of_fungi/methodsinantifungalsusceptibilitytesting/susceptibility_testing_of_yeasts/; last access date December 13, 2018.

14. J. Vlainić, I. Kosalec, K. Pavić, D. Hadjipavlou-Litina, E. Pontiki and B. Zorc, Insights into biological activity of ureidoamides with primaquine and amino acid moieties, J. Enzyme Inhib. Med. Chem. 33 (2018) 376-382; https://doi.org/10.1080/14756366.2017.1423067

15. S. Purser, P. R. Moore, S. Swallow and V. Gouverneur, Fluorine in medicinal chemistry, Chem. Soc. Rev. 37 (2008) 320-330; https://doi.org/10.1039/b610213c 